\title{
A IMPORTÂNCIA DOS MEDICAMENTOS GENÉRICOS PARA OS IDOSOS
}

\author{
Patrícia Martins Fernandes Oliveira ${ }^{1}$ \\ Leonardo Guimaraes de Andrade ${ }^{2}$
}

RESUMO: Medicamentos são componentes indispensáveis para o tratamento de várias doenças, possibilitando a reabilitação e manutenção da saúde. A Organização Mundial de Saúde (OMS) tem publicado bianualmente desde 1977 sua lista de "Medicamentos Essenciais”. Essa lista central apresenta um conjunto mínimo de medicamentos necessários para um sistema básico de saúde, enumerando aqueles que são mais eficazes, seguros e econômicos para doenças prioritárias. Medicamentos genéricos são aqueles cuja patente original expirou e que agora podem ser produzidos por outros fabricantes que não a empresa inovadora original (detentora da patente). Diversos estudos demonstraram que existe uma resistência por parte dos médicos e por consequência dos idosos em buscar por medicações genéricas. Ainda que o valor dos medicamentos prescritos e dos genéricos sejam divergentes e muitas vezes mais caros. O que torna o aceite por parte da população idosa dos produtos genéricos se tornando um desafio ao mercado farmacêutico. Assim este trabalho tem como objetivo principal compreender a importância do consumo de medicamentos genéricos para os idosos. Assim como demonstrar quais as Políticas de Saúde existentes referentes à Assistência farmacêutica e sua efetivação na Saúde Básica de Saúde e; Identificar as formas de obtenção de medicamentos incluindo o SUS e o Programa Farmácia Popular. O estudo se baseará em uma revisão bibliográfica, uma vez que se pretende reunir as informações já encontradas sobre o assunto, e será aplicado uma metodologia qualitativa, com foco no caráter subjetivo da bibliografia analisada, por conceitos, definições, posições e opiniões, bem como uma metodologia dedutiva e descritiva.

Palavras- Chaves: Medicamentos Genéricos. Idosos. Farmácia Popular.

ABSTRACT: Medicines are essential components for the treatment of various diseases, enabling rehabilitation and health maintenance. The World Health Organization (WHO) has been publishing its list of "Essential Medicines" biannually since 1977. This central list presents a minimum set of drugs needed by a basic health system, listing those that are most effective, safe and economical for priority diseases. Generic drugs are drugs whose original patent has expired and which can now be produced by manufacturers other than the original innovator (patent holder). Several studies have shown that there is resistance on the part of physicians and, consequently, of the elderly to seek generic medications. Even though the value of prescription drugs and generics are divergent and often more expensive. What

\footnotetext{
r Discente do curso de farmácia pela UNIG.E-mail: fernandesoliveirar829@gmail.com

${ }^{2}$ Professor e orientador do curso de farmácia da UNIG.
} 
makes the acceptance by the elderly population of generic products becoming a challenge to the pharmaceutical market. Thus, this work has as its main objective to understand the importance of the consumption of generic drugs for the elderly. As well as demonstrating what the existing Health Policies regarding pharmaceutical assistance and its effectiveness in Basic Health and; Identify ways to obtain medicines including the SUS and the Popular Pharmacy Program. The study will be based on a literature review, as it intends to gather the information already found on the subject, and a qualitative methodology will be applied, focusing on the subjective character of the analyzed bibliography, by concepts, definitions, positions and opinions, as well as a deductive and descriptive methodology.

Keywords: Generic Medicines. Seniors. Popular Pharmacy.

\section{INTRODUÇÃO}

Medicamentos são componentes indispensáveis para o tratamento de várias doenças, possibilitando a reabilitação e manutenção da saúde (DE AMORIM RODRIGUES et al., 2020). A Organização Mundial de Saúde (OMS) tem publicado bianualmente desde 1977 sua lista de "Medicamentos Essenciais". Essa lista central apresenta um conjunto mínimo de medicamentos necessários para um sistema básico de saúde, enumerando aqueles que são mais eficazes, seguros e econômicos para doenças prioritárias. Essas condições prioritárias são selecionadas com base na relevância atual e futuro estimado para a saúde pública e no potencial para tratamento seguro e com boa relação custo-benefício. As versões mais

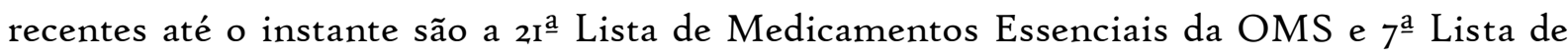
Medicamentos Essenciais da OMS para Crianças (OMS, 2019). No entanto, o acesso a medicamentos foi uma dificuldade enfrentada em vários países durante muitas décadas, afetando cerca de um terço da população mundial (BLATT et al., 2012; NIELSON et al., 2018).

Os custos elevados para aquisição de medicamentos eram relativos a um processo produtivo dispendioso, associado a inúmeras exigências para regulamentação dos mesmos junto aos órgãos sanitários (BLATT et al., 2012; NIELSON et al., 2018). Assim, a maioria dos medicamentos presentes na lista da OMS já não possuem patente em vigência e estão disponíveis como produtos genéricos, que muitas vezes são oferecidos a preços mais baixos do que o medicamento de referência, potencialmente reduzindo custos para os pacientes e o sistema de saúde. Nesse cenário, a utilização de medicamentos genéricos é incentivada, a fim de reduzir custos e aumentar o acesso à saúde (DAVE et al., 2017). 
Medicamentos genéricos são aqueles cuja patente original expirou e que agora podem ser produzidos por outros fabricantes que não a empresa inovadora original (detentora da patente) (DUNNE; DUNNE, 2015). O uso de produtos farmacêuticos genéricos representa mais da metade do volume total de produtos dessa indústria utilizados em todo o mundo, mas apenas $18 \%$ do valor total do mercado farmacêutico. Essas proporções variam por região e país, mas o consumo de medicamentos genéricos é consistentemente superior aos medicamentos de referência na maioria dos países, sendo uma das tecnologias de saúde mais utilizadas no mundo (DAVE et al., 2017).

Essas duas leis federais permitiram que o segmento de medicamentos genéricos no Brasil tomasse forma semelhante ao que já era experimentado em outros países, facilitando o acesso ao tratamento medicamentoso para a população de baixa renda (BOEIRA, 20II). Assim, desde o início da implementação dos genéricos no mercado brasileiro, entre os anos de 2000 e 2003 , estes produtos já possuíam preços cerca de $40 \%$ menores que seus correspondentes de referência (DE OLIVEIRA LEMES et al., 2018).

Diversos estudos demonstraram que existe uma resistência por parte dos médicos e por consequência dos idosos em buscar por medicações genéricas. Ainda que o valor dos medicamentos prescritos e dos genéricos sejam divergentes e muitas vezes mais caros. $\mathrm{O}$ que torna o aceite por parte da população idosa dos produtos genéricos se tornando um desafio ao mercado farmacêutico.

\section{OBJETIVOS \\ 2.1 Objetivo Geral}

Desta maneira, o objetivo geral deste trabalho busca compreender a importância do consumo de medicamentos genéricos para os idosos.

\subsection{Objetivos Específicos}

Para atingir o objetivo principal devemos:

- Demonstrar quais as Políticas de Saúde existentes referentes à Assistência farmacêutica e sua efetivação na Saúde Básica de Saúde.

- Identificar as formas de obtenção de medicamentos incluindo o SUS e o Programa Farmácia Popular. 


\section{METOdOLOGIA}

O estudo se baseará em uma revisão bibliográfica, uma vez que se pretende reunir as informações já encontradas sobre o assunto, e será aplicado uma metodologia qualitativa, com foco no caráter subjetivo da bibliografia analisada, por conceitos, definições, posições e opiniões, bem como uma metodologia dedutiva e descritiva.

Os procedimentos para a revisão da literatura e a construção do embasamento teórico, levando em consideração ensinamentos de diversos autores, diversos anos e perspectivas, e será desenvolvimento nas seguintes etapas: escolha do tema e delimitação do tema; levantamento bibliográfico preliminar; elaboração do plano provisório de assunto; busca das fontes; leitura do material; fichamento; organização lógica do assunto; e por fim redação do texto.

\section{REFERENCIAL TEÓRICO}

O ser humano passa por diversas fases ao longo da vida, nasce e adquire consciência à medida que tem experiências que o modelam e constroem sua personalidade. Essas fases vão do nascimento até a morte do indivíduo e cada período é marcado por diversas especificidades. A velhice é o último estágio do ciclo vital e ao longo dos anos têm ganhado atenção e estudos mais específicos, já que, como consequência dos avanços tecnológicos na saúde, o homem tem vivido cada dia por mais anos.

Segundo Rabêlo e Nascimento (2007) a modernidade favoreceu o aumento significativo na expectativa de vida, na medida em que proporcionou condições mais favoráveis para um envelhecimento ativo. Nesta etapa da vida, ocorrem significativas mudanças físicas, biológicas e funcionais, nota-se um declínio natural das funções vitais e cognitivas e maior suscetibilidade a condições de fragilidade e doença. Consequentemente, pode ocorrer uma gradativa diminuição na autonomia e no autocuidado. Em países desenvolvidos são considerados "idosos jovens" pessoas entre 65 e 74 anos, a partir dos 75 até os 84 são os “idosos" e por fim, são considerados idosos mais velhos, aqueles com 85 anos ou mais e que não possuem autonomia para as atividades diárias (PAPALIA; FELDMAN, 2013).

Entretanto, em países subdesenvolvidos, como é o caso do Brasil, são considerados idosos pessoas acima de 60 anos, tendo seus direitos assegurados pela Lei $n^{\circ} 8.842 / 94$. Torna- 
se clara a associação entre longevidade e desenvolvimento de uma sociedade graças a melhorias na nutrição e cuidados de saúde em geral, com isso, a população com Ioo anos ou mais está aumentando notavelmente (KINSELLA; HE, 2009).

Existem doenças crônicas que afetam a população idosa e que alteram suas capacidades funcionais. As Doenças Crônicas Não Transmissíveis (DCNT) mais comuns são as doenças cardiovasculares, os cânceres, as doenças respiratórias crônicas, o diabetes mellitus e os transtornos psiquiátricos. Essas doenças requerem acompanhamento constante, pois, em razão de sua natureza, não têm cura (BRASIL, 2006a).

\section{I Os Medicamentos Genéricos}

O mercado farmacêutico se caracteriza por sua alta complexidade devido à quantidade de atores envolvidos desde o processo de produção até a prescrição. São bens de consumo, que do ponto de vista econômico apresentam algumas peculiaridades que os tornam específicos e, como bens para a saúde, constituem o recurso médico e terapêutico mais utilizado. Isso significa que as limitações na sua produção, circulação e consumo podem ter um impacto negativo significativo na saúde da população e na distribuição das reservas de saúde na sociedade (CAMPOS, 2018).

Nos Estados Unidos, a Lei Federal de Concorrência de Preços de Medicamentos e Restauração de Termos de Patentes, informalmente conhecida como Lei Hatch-Waxman, sancionada em 1984, foi um marco para o mercado farmacêutico americano ao encurtar os prazos de registros de medicamentos cujas patentes haviam expirado, acelerando a entrada desses medicamentos no mercado em sua forma genérica. Isso permitiu uma redução drástica nos preços praticados e impulsionou o segmento dos genéricos, fato que ampliou o acesso à população de mais baixa renda a vários medicamentos (BOEIRA, 20II).

Depois do pioneirismo do Estados Unidos quanto a regulamentação dos medicamentos genéricos, outras nações acompanharam a tendência e também legislaram sobre a matérias e se tornaram assim líderes no segmento de genéricos como Canadá, Alemanha, Japão, Grã-Bretanha, Dinamarca e Holanda, representando assim cerca de 60\% do mercado mundial de genéricos (NIELSON et al., 2018).

O mercado internacional passou por grandes transformações desde os anos 90 através concentração industrial, excelentes margens de lucros, aumento no consumo de 
medicamentos, a elevação de preços e a pressão competitiva por parte dos genéricos. Nesse cenário, observou-se uma retração no consumo de medicamentos por parte da Europa, enquanto Estados Unidos e América Latina intensificaram sua participação no mercado (BOEIRA, 20II).

Para que um novo medicamento seja formulado e possa ser comercializado, é necessária a realização de pesquisas para descoberta do princípio ativo, desenvolvimento de um processo de formulação viável do ponto de vista industrial e que seja comercialmente justificado. Isso exige da indústria farmacêutica alto investimento financeiro em todas as etapas, desde o desenvolvimento até a inserção do produto no mercado. Soma-se ao custo financeiro o gasto de tempo na execução dos testes exigidos, que nem sempre garantem o sucesso do desenvolvimento do novo medicamento. Isso acarreta custos de até bilhões de dólares para desenvolver produtos inovadores. No entanto, os medicamentos recémdesenvolvidos são protegidos pelas patentes (PONTES, 2017; JESUS, 2018). A exclusividade temporária no mercado garantida pelas patentes permite a empresas desenvolvedoras de medicamentos inovadores recuperar os gastos no setor de P\&D e lucrar até que a patente expire e a indústria passe a enfrentar a competição de medicamentos genéricos e similares (BOEIRA, 20II; PONTES, 2017).

Nesse cenário, a indústria farmacêutica dos últimos anos tem se apoiado na evolução tecnológica dos conhecimentos químicos e farmacológicos para investir no desenvolvimento de novos medicamentos. O patamar da indústria farmacêutica atual é caracterizado pela liderança de multinacionais atuantes no mercado que investem grandes quantias em seus departamentos de pesquisa e desenvolvimento ( $P \& D)$ e marketing para se diferenciarem frente a concorrência. $O$ foco na pesquisa e desenvolvimento visa principalmente a obtenção de patentes. As patentes correspondem a ativos valiosos visto que são importante instrumento competitivo no mercado, garantem o domínio da exploração monopolística de um produto ou processo, impedindo a entrada de concorrentes (BOEIRA, 20II; PONTES, 2017). No entanto, todos estes gastos relacionados ao desenvolvimento e comercialização de medicamentos de referência fazem com estes se apresentem em preços maiores que os genéricos correspondentes (DE AMORIM RODRIGUES et al., 2020).

Em 2015, por exemplo, foi observado um crescimento do mercado farmacêutico a nível global de 12,2\% em relação ao ano de 2014. Esse crescimento tem por base criação de 
novos medicamentos, diminuição de preços de medicamentos de referência e o maior consumo de medicamentos genéricos que representou um gasto de \$7,9 bilhões em 2015 (MAIRA; DAMIN; ZILBER, 2017).

O que define um medicamento genérico é ser um produto farmacêutico a base do mesmo princípio ativo e excipientes, forma farmacêutica, concentração, via de administração e indicação de uso que seu correspondente de referência. Os genéricos devem ser capazes de apresentar biodisponibilidade e bioequivalência semelhante aos medicamentos de referência, se comportando no organismo de forma semelhante. Para isso, devem passar por testes de segurança, de eficácia e de qualidade, assim como os medicamentos de referências são submetidos (DE OLIVEIRA LEMES et al., 2018).

Os medicamentos genéricos possuem como diferenciais no mercado farmacêutico com preços mais acessíveis por uma série de fatores como menores investimentos em marketing, a ausência de gastos com pesquisa e desenvolvimento e ensaios clínicos necessários para o registro de produto inovador e a maior competição entre as indústrias farmacêuticas que produzem tanto o medicamento de referência quanto seus concorrentes e correspondentes genéricos respectivamente (DE AMORIM RODRIGUES et al., 2020). Assim, o mercado conta nos últimos anos com medicamentos genéricos validos para mais de 95\% das enfermidades conhecidas (NIELSON et al., 2018).

Nesse sentido, os genéricos se traduzem em uma economia considerável para o Estado. Por exemplo, nos Estados Unidos eles representam cerca de 70\% do número total de prescrições e menos de $20 \%$ do custo total dos medicamentos prescritos. Na Europa, a média dos genéricos ronda os $40 \%$ e, em 2007, o Reino Unido poupou cerca de 6 bilhões de euros graças à receita dos genéricos, enquanto na Espanha a economia foi de apenas cerca de 125 milhões de euros (CAMPOS, 2018).

\subsection{Políticas de saúde referentes à assistência farmacêutica}

No ano de 2003 foi realizada a I Conferência Nacional de Medicamentos e Assistência Farmacêutica, que fundamentaram para que em 2004 o Conselho Nacional de Saúde (CNS) aprovar a Política Nacional de Assistência farmacêutica o PNAF. Sendo definida como:

\footnotetext{
Um conjunto de ações voltadas à promoção, proteção e recuperação da saúde, tanto individual como coletiva, tendo o medicamento como insumo essencial e visando o acesso e seu uso racional. Este conjunto envolve a pesquisa, o desenvolvimento e a produção de medicamentos e insumos, bem como a sua seleção, programação, aquisição, distribuição, dispensação, garantia da qualidade dos produtos e serviços,
} 
acompanhamento e avaliação de sua utilização, na perspectiva da obtenção de resultados concretos e da melhoria da qualidade de vida da população (BRASIL, 2004).

Assim de acordo com a PNAF, a Assistência Farmacêutica é a principal política pública que orienta a formulação de políticas setoriais. Com eixos estratégicos, manutenção, qualificação dos serviços de assistência farmacêutica na rede pública e a qualificação em recursos humanos, assim como a descentralização das ações (BRASIL, 2004).

Assim buscando assegurar que a população tenha acesso a medicamentos seguros, eficientes e de qualidade, com pouco custo, os gestores do SUS buscam por meio de algumas diretrizes tornar real esse acesso (BRASIL,200I).

\subsubsection{Adoção de relação de medicamentos essenciais}

Vão fazer parte desta relação os medicamentos considerados essenciais, os básicos e indispensáveis para o atendimento de grande parte dos problemas de saúde da população. Estes medicamentos precisam de disponibilidade contínua para a população, e vão compor uma relação nacional de referência estrutural para a produção farmacêutica (BRASIL,20oI).

\subsubsection{Regulamentação sanitária de medicamentos}

Aqui são enfatizadas as questões vinculadas ao registro de medicamentos, sua autorização para o funcionamento de empresas e estabelecimentos, assim como restrições ao uso de medicamentos que possam ser inadequados. A promoção do uso de medicamentos genéricos, neste ponto ganha uma atenção especial uma vez que ela possibilita a consolidação do uso dos genéricos, como:

a) a obrigatoriedade da adoção da denominação genérica nos editais, propostas, contratos e notas fiscais - bem como de exigências sobre requisitos de qualidade dos produtos;

b) a obrigatoriedade da adoção da denominação genérica nas compras e licitações públicas de medicamentos realizadas pela Administração Pública;

c) a adoção de exigências específicas para o aviamento de receita médica ou odontológica, relativas à sua forma e à identificação do paciente e o profissional que a prescreve;

d) a apresentação da denominação genérica nas embalagens, rótulos, bulas, prospectos, textos e demais materiais de divulgação e informação médica (BRASIL,2001, p.14).

\subsubsection{Reorientação da assistência farmacêutica}

Vem a ser um modelo de assistência farmacêutica de forma a reorientar e que não exista restrição à aquisição e distribuição de medicamentos. As políticas incluídas neste 
tópico, procuram implementar em três esferas do SUS, as atividades vinculadas à promoção do acesso da população aos medicamentos essenciais (BRASIL,200I).

\subsubsection{Promoção do uso racional de medicamentos}

Existe uma atenção especial em relação a informação relativa às repercussões sociais e econômicas do receituário médico, em especial ao nível ambulatorial, no tratamento de doenças prevalentes. São realizadas medidas educacionais dos usuários ou consumidores sobre o risco da automedicação, interrupção e da troca da medicação prescrita, assim como a demanda da receita médica em relação à dispensão de medicamentos tarjados. De forma paralela, estas questões possuem a finalidade de desenvolver atividades dirigidas aos profissionais prescritores dos produtos e aos dispensadores (BRASIL,200I).

\section{DISCUSSÃO}

Como discorrido ao longo do trabalho, os principais consumidores de medicamentos genéricos é a população idosa. Aproximadamente 80\% desta população faz uso de ao menos um medicamento ao dia, e um terço deste número acaba consumindo no mínimo cinco medicamentos de forma simultânea, tornando-os a maior fatia consumidora do mercado farmacêutico.

Ainda que os medicamentos genéricos tenham um melhor custo/benefício, existe uma visão negativa destes medicamentos por pessoas de menor renda e idosas. Populações que demandam uma necessidade maior de que os medicamentos possuam seus valores reduzidos. Os idosos em grande maioria apresentam doenças crônicas e fazem uso de um grande número de medicamentos, que geram um impacto em sua renda.

Motivos que tornam essenciais que os médicos prescrevam medicamentos genéricos, visando melhor custo aos idosos e assim a manutenção de sua saúde.

\section{CONCLUSÃO}

Os medicamentos genéricos são uma conquista para a promoção da saúde da população mundial, principalmente de baixa renda. Os menores preços associados aos genéricos se relacionam com a não necessidade da realização de pesquisa e desenvolvimento, dos estudos clínicos e do marketing exigido para o registro e comércio de um produto inovador. 
Nesse sentido, a população precisa receber as informações corretas e precisas sobre a relevância e eficiência dos medicamentos genéricos em relação aos de referência. Eles permitem o mesmo tratamento que os medicamentos de referência e com preços mais acessíveis, diminuído os gastos finais com saúde entre as famílias, principalmente para aquelas com pessoas idosas.

O uso de medicamentos entre os idosos, é essencial como forma terapêutica de compensação das mudanças sofridas no processo de envelhecimento. Aumentado ainda mais a importância de um uso racional de medicamentos, por meio de uma boa assistência farmacêutica. Ainda que receitados pela assistência básica de saúde, os medicamentos genéricos ainda não são utilizados de forma frequente pelos idosos.

Como vimos há uma mistificação com relação a estas medicações, motivo o qual muitos idosos preferem comprar pela farmacêutica tradicional ao invés dos medicamentos genéricos. Que são desenvolvidos e comercializados com valores acessíveis, o que não pesaria no bolso destes pacientes, cuja renda acaba prejudicada pelo grande uso de medicamentos.

\section{REFERÊNCIAS}

BRASIL. Lei no 9.279, de 14 de maio de 1996. Regula direitos e obrigações relativos à propriedade industrial. Diário Oficial [da] República Federativa do Brasil, Brasília, DF, I4 mai. 1996.

BRASIL. Lei no 9.787, de io de fevereiro de 1999. Altera a Lei no 6.36o, de 23 de setembro de 1976, que dispõe sobre a vigilância sanitária, estabelece o medicamento genérico, dispõe sobre a utilização de nomes genéricos em produtos farmacêuticos e dá outras providências. Diário Oficial [da] República Federativa do Brasil, Brasília, DF, ıo mai. 1999.

BRASIL. Ministério da Saúde. Secretaria de Políticas de Saúde. Departamento de Atenção Básica. Política nacional de medicamentos 200I/Ministério da Saúde, Secretaria de Políticas de Saúde, Departamento de Atenção Básica. - Brasília: Ministério da Saúde, 200 .

BRASIL. Conselho Nacional de Saúde. Resolução n. 338, de o6 de maio de 2004. Aprova a Política Nacional de Assistência Farmacêutica. Diário Oficial da União, Poder Executivo, Seção I n. 96, 20 de maio de 2004. Brasília: Ministério da Saúde, 2004.

DAVE, Chintan V. et al. High generic drug prices and market competition: a retrospective cohort study. Annals of internal medicine, v. 167, n. 3, p. 145-151, 2017. 
DE AMORIM RODRIGUES, Larice et al. Medicamentos genéricos nos últimos 20 anos e a percepção dos consumidores. Revista Saúde dos Vales; ISSN: 2674-8584 V.I, n.I. 2020.

DE OLIVEIRA LEMES, Erick et al. História do Medicamento Genérico no Brasil. Ensaios e Ciência C Biológicas Agrárias e da Saúde, v. 22, n. 2, p. I19-I23, 2018.

DUNNE, Suzanne S.; DUNNE, Colum P. What do people really think of generic medicines? A systematic review and critical appraisal of literature on stakeholder perceptions of generic drugs. BMC medicine, v. 13, n. I, p. I-27, 2015.

NIELSON, Sylvia Escher de Oliveira et al. A importância dos medicamentos genéricos no brasil. Revista de Trabalhos Acadêmicos-Universo-Goiânia, n. 4, 2018.

OMS (Organização Mundial da Saúde). WHO Model Lists of Essential Medicines. 2019. Disponível em: https://www.who.int/groups/expert-committee-on-selection-and-use-ofessential-medicines/essential-medicines-lists; Acesso em o9 de abril de 2021. 